\title{
Incompressible models of magnetohydrodynamic Richtmyer-Meshkov instability in cylindrical geometry
}

\author{
A. Bakhsh* \\ Applied Mathematics and Computational Sciences, CEMSE Division, KAUST, Saudi Arabia \\ R. Samtaney \\ Mechanical Engineering, PSE Division, KAUST, Saudi Arabia
}

(Received 17 November 2018; published 27 June 2019)

\begin{abstract}
The Richtmyer-Meshkov instability (RMI) occurs when a shock impulsively accelerates an interface between two different fluids, and it is important in many technological applications such as inertial confinement fusion (ICF) and astrophysical phenomena such as supernova. Here, we present incompressible models of an impulsively accelerated interface separating conducting fluids of different densities in cylindrical geometry. The present study complements earlier investigations on linear and nonlinear simulations of RMI. We investigate the influence of a normal or an azimuthal magnetic field on the growth rate of the interface. This is accomplished by solving the linearized initial value problem using numerical inverse Laplace transform. For a finite normal magnetic field, although the initial growth rate of the interface is unaffected by the presence of the magnetic field, at late-time the growth rate of the interface decays. This occurs by transporting the vorticity by two Alfvén fronts which propagate away from the interface. For the azimuthal magnetic field configuration, the suppression mechanism is associated with the interference of two waves propagating parallel and antiparallel to the interface that transport vorticity and cause the growth rate to oscillate in time with nearly a zero mean value. Comparing the results of the incompressible models with linear compressible MHD simulations show reasonable agreement at early time of simulations.
\end{abstract}

DOI: 10.1103/PhysRevFluids.4.063906

\section{INTRODUCTION}

The Richtmyer-Meshkov instability (RMI) occurs when a shock wave impulsively accelerates a perturbed density interface separating two different fluids [1,2]. The RMI occurs in a variety of physical applications. It is important in astrophysical phenomena such as supernova explosions, supersonic combustion in hypersonic air-breathing engines, and inertial confinement fusion (ICF) [3-5]. The linearized perturbations equations, for the case of a reflected shock wave, were first derived for general fluids by Richtmyer (1960), who obtained their numerical solution for perfect gases. RMI may not be classified as a classical hydrodynamic instability with modal analysis because the perturbations grow algebraically (linearly in time) rather than exponentially. Fraley [6] used Laplace transforms in time to obtain an analytical solution for the asymptotic linear growth rate for the same problem as Richtmyer. He also found that the pressure perturbations across the interface directly related to the vorticity that initially deposited on the interface. The RMI in the presence of a magnetic field initially oriented parallel to the incident shock direction has been investigated numerically and theoretically. Nonlinear ideal magnetohydrodynamics (MHD) simulations of a

\footnotetext{
*Present address: Mathematical Sciences, AS College, UQU, Saudi Arabia.
} 
shock interaction with an oblique planar contact discontinuity were discussed by Samtaney [7]. It was demonstrated that the RMI is suppressed by the existence of such a magnetic field. The primary suppression mechanism may be attributed to transport of baroclinic vorticity away from the density interface by slow- or intermediate mode shocks transporting the vorticity away from the interface. This results were reconfirmed in the context of incompressible MHD linear stability analysis by Wheatley et al. [8]. They demonstrated that vorticity is transported away from the interface by Alfvén fronts. More detailed nonlinear MHD simulations extending the parameter ranges of earlier investigations demonstrated the suppression of RMI [9]. The effect of transverse magnetic field, oriented parallel to the interface, recently has been investigated analytically and numerically [10-13]. In fact, in a super-conductive plasma, the existence of a transverse magnetic field behaves in a fashion somewhat similar to surface tension on the interface. The transverse magnetic field inhibits the growth of surface perturbations and as a result, stabilizes the instability [10]. Cao et al. [11] studied this case in the framework of incompressible media through an analytical development of a magnetized impulsive model. They also found an inhibition of the RMI growth with oscillations of the perturbation amplitude. Wheatley et al. [13] investigated nonlinear compressible simulations and incompressible model of MHD-RMI for the case of a transverse magnetic field, i.e., there was no normal component of the magnetic field at the interface and found oscillatory solutions of the growth rates with the RMI still being suppressed. One of the early investigations of hydrodynamic RMI in cylindrical geometry was carried out by Zhang and Graham [14], in which the imploding and exploding shocks were examined for positive and negative Atwood ratio interfaces, which is defined as $A=\left(\rho_{2}-\rho_{1}\right) /\left(\rho_{2}+\rho_{1}\right)$, where $\rho_{1}, \rho_{2}$ are the densities of the fluids under investigation. The stability of density interface was studied by Plesset [15] for spherical bubbles $\left(\rho_{2} \ll \rho_{1}\right)$. Plesset's linear analysis was extended to the spherical system composing $\mathrm{N}$ concentric fluid shells by Mikaelian [16] where he investigated the linear RMI and RTI of incompressible flow. Mikaelian treated the linear regime by assuming that the perturbations are small. In his work, a formulated model to estimate the mixing layer width between different fluids was presented. Likewise, he also investigated the linear stability of RTI and RMI in concentric cylindrical shells. He discussed the freeze-out phenomena which leads to a zero asymptotic growth rate via numerical simulations and developed a simple model for the evolution of turbulent mix [17]. Lombardini and Pullin [18] investigated the RMI in cylindrical and spherical geometries with azimuthal and axial perturbations and developed a linear incompressible theory for the case of an imploding or exploding shock. In particular, they developed a theory for the asymptotic growth rate of perturbations and further derived a unified expression for the asymptotic impulsive growth rate in planar, cylindrical and spherical cases. In their work, the linear analysis was complemented and verified by compressible nonlinear simulations with a small amplitude perturbation. In converging geometry, the RMI is typically followed by the Rayleigh-Taylor instability (RTI) due to the continued acceleration or deceleration of the interface towards the center of convergence, depending on sign of Atwood number, which was not captured by the model of Lombardini and Pullin. The physical configuration that is relevant to ICF is one where the main incident shock is a converging shock. So it is of interest to examine the RM instability in a converging geometry, especially in the presence of a magnetic field and investigate the physical mechanisms of suppression if any. We aim to understand the effect of a magnetic field on RMI of sinusoidally perturbed density interface separating incompressible conducting fluids. Richtmyer proposed that, by the time the transmitted and reflected waves have traveled a long distance from the interface compared to the wavelength of the perturbation, the subsequent motion at the interface can be considered incompressible [19]. Therefore, we propose an incompressible model that is computationally inexpensive compared with the nonlinear and linear simulations wherein a full system of hyperbolic PDE is solved numerically. It is also useful as a model of the early linear stages of RMI. We consider two cases: (a) the magnetic field is oriented parallel to the motion of the shock and (b) the case of a magnetic field parallel to the mean interface location. The initial condition for this flow and the setup for the model problem are illustrated in Figs. 1 and 2 for each case respectively in Sec. II. We use an approach similar to the one presented by Wheatley et al. $[8,13]$ so that the interface motion occurs only due to the impulsive acceleration. 


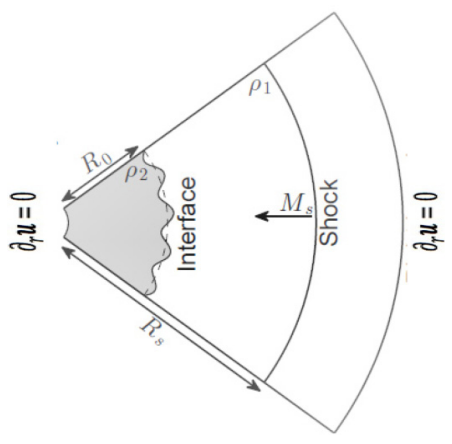

(a)

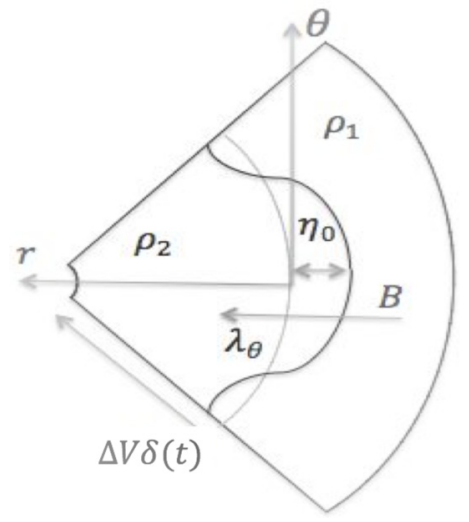

(b)

FIG. 1. (a) Initial condition for the shock driven compressible MHD RMI (b) Initial condition for the corresponding impulse driven, incompressible model.

In Sec. III, the ideal incompressible MHD equations are linearized and the resulting initial value problem is presented. The solutions of the proposed models are obtained using a numerical method that presented in Appendix A 1. The behavior predicted by these models are compared with the results of the shock-driven simulations of the linearized compressible ideal MHD equations. These results are displayed in Sec. IV.

\section{INCOMPRESSIBLE MODEL FORMULATION}

In this section, we present incompressible models for the MHD RMI for two cases: the first one in which the magnetic field orientation is normal to the interface and second where the field is parallel to the interface. In this section, we present the base or background state about which the

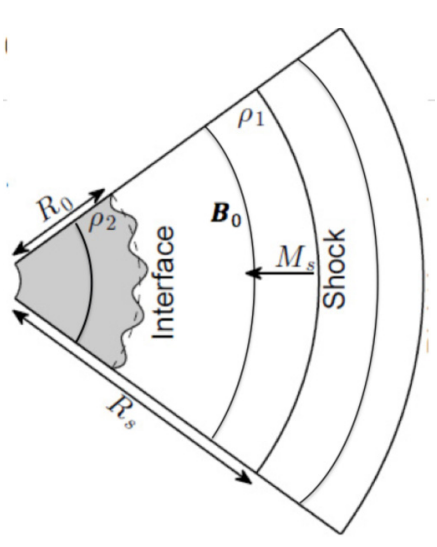

(a)

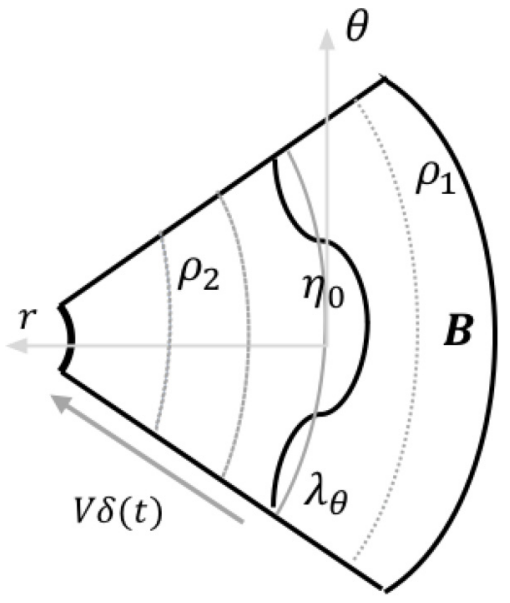

(b)

FIG. 2. (a) Initial condition for the shock driven compressible MHD RMI (b) Initial condition for the corresponding impulse driven, incompressible model. 
linearization is performed. The linear equations governing the perturbation quantities are presented in detail in Sec. III.

\section{A. Normal magnetic field}

In this case, the magnetic field is oriented in the direction of the shock motion normal to the contact discontinuity (CD) between two fluids with different densities, $\rho_{1}, \rho_{2}$. Figure 1(a) illustrates the initial condition for a flow characterized by the density ratio across the $\mathrm{CD}, \rho_{2} / \rho_{1}$, the ratio of specific heats $\gamma$, the incident shock of Mach number $M$ and the nondimensional strength of the magnetic field, $\beta=2 \stackrel{\circ}{p} / \stackrel{B}{ }^{2}$, where $\stackrel{\circ}{p}$ is the initial pressure in the unshocked regions of the flow and $\stackrel{B}{ }$ is the magnitude of the magnetic field. The model for this flow is shown in Fig. 1(b), where the shock is replaced by an impulsive acceleration in $r$-direction, $\Delta V \delta(t)$, where $\delta(t)$ is the Dirac delta function in time. The incompressible ideal MHD equations in cylindrical coordinates are linearized about a base flow (denoted with a $\left\{{ }^{\circ}\right\}$ ) that results from the impulsive acceleration of an unperturbed interface. This base flow has no $\theta$ dependence and zero azimuthal velocity $u_{\theta}$. Our choice of reference frame is attached to the impulsively started contact discontinuity which results in the mean radial velocity $\left(\stackrel{\circ}{u}_{r}\right)$ at the contact being zero for all time. The base flow is governed by the following equations:

$$
\begin{aligned}
\stackrel{\circ}{\rho}(r) & =\stackrel{\circ}{\rho}_{1}+H\left(r-R_{0}\right)\left(\stackrel{\circ}{\rho}_{2}-\stackrel{\circ}{\rho}_{1}\right), \quad \stackrel{\circ}{u}_{r}=0, \quad \stackrel{\circ}{u}_{\theta}=0, \quad \stackrel{\circ}{B}_{r}=b_{r}, \quad \stackrel{\circ}{B}_{\theta}=0, \\
\stackrel{\circ}{p}(r, t) & =-\stackrel{\circ}{\rho}_{1} \Delta V \delta(t) r-H\left(r-R_{0}\right)\left(\stackrel{\circ}{\rho}_{2}-\stackrel{\circ}{\rho}_{1}\right) \Delta V \delta(t) r,
\end{aligned}
$$

where $\rho$ is the density, $p$ is the pressure, $\mathbf{u}$ is the velocity, and $\mathbf{B}$ is the magnetic field with initial constant magnitude $b_{r}$ in the normal direction to the interface, $H\left(r-R_{0}\right)$ is the Heaviside function and $R_{0}$ is the initial position of the interface.

When the interface is perturbed, the density becomes $\stackrel{\circ}{\rho}(r-f)$, where $f(\theta, t)$ is the location of the interface and $f \ll \lambda_{\theta}$, where $\lambda_{\theta}$ is the wavelength. An important point must be clarified here: for a purely radial flow, the velocity must have a $\frac{1}{r}$ dependence. Therefore, the base flow is an approximate solution to the governing equations that is valid while the interface is at large radius. In this case, large radius implies that over the time period of interest, the change in the base flow velocity (proportional to $\frac{1}{r(t)}-\frac{1}{R_{0}}$ ) is small compared to the velocity perturbations. This assumption is also applied for the magnetic field which has $\frac{1}{r}$ dependence. The model then accounts for the early time evolution of the perturbations in cylindrical coordinates due to the radial impulsive acceleration. Hence, we assume the interface moves with a constant impulsive speed of the shocked contact discontinuity; this will prevent the deceleration effect of cylindrical geometry on the interface as it converges to the origin. As previously noted in Bakhsh et al. [20], Rayleigh-Taylor instability (RTI) is manifested during the deceleration phase; and for positive Atwood ratio this results in a phase inversion. As a result of the preceeding assumptions, the RTI phase of the instability is not captured by our model.

\section{B. Azimuthal magnetic field}

This section presents an incompressible model of the MHD RMI for a magnetic field parallel to the mean interface location. The initial condition for a shocked sinusoidally perturbed density interface is shown in Fig. 2(a), in the presence of an azimuthal magnetic field $\mathbf{B}$. The corresponding setup for the incompressible model is shown in Fig. 2(b). An impulsive acceleration in $r$-direction, $\Delta V \delta(t)$, is used to replace the shock acceleration of uniform conducting fluids of different densities $\rho_{1}$ and $\rho_{2}$. The initial magnetic field is aligned with the mean interface location in $\theta$-direction with the nondimensional strength $\beta=\stackrel{p}{p} / \stackrel{\circ}{ }^{2}$. The base flow is governed by the following equations:

$$
\begin{aligned}
\stackrel{\circ}{\rho}(r) & =\stackrel{\circ}{\rho}_{1}+H\left(r-R_{0}\right)\left(\stackrel{\circ}{\rho}_{2}-\stackrel{\circ}{\rho}_{1}\right), \quad \stackrel{\circ}{u}_{r}=0, \quad \stackrel{\circ}{\theta}_{\theta}=0, \\
\stackrel{\circ}{B}_{r} & =0, \quad \stackrel{\circ}{B}_{\theta}=b_{t}, \quad \stackrel{\circ}{p}(r, t)=\stackrel{\circ}{p},
\end{aligned}
$$


where $b_{t}$ is the initial magnitude of the magnetic field in the azimuthal direction. We linearize the ideal MHD equations about this flow.

\section{LINEAR STABILITY ANALYSIS}

We seek solutions to the model problem that satisfy the linearized equations of ideal incompressible MHD. The perturbed equations are linearized about the base flow by setting the density equal to

$$
\rho(r, \theta, t)=\stackrel{\circ}{\rho}_{1}+H[r-f(\theta, t)]\left(\stackrel{\circ}{\rho}_{2}-\stackrel{\circ}{\rho}_{1}\right),
$$

where $f(\theta, t)=\eta(t) e^{i m \theta} \ll \lambda_{\theta}$, where $\eta(t)$ is the amplitude of the perturbed interface, $m$ is an azimuthal perturbation wave number and by assuming that all other flow quantities have the form $q^{\prime}(r, \theta, t)=\stackrel{q}{q}(r)+q^{\prime}(r, \theta, t)$, where $q^{\prime}$ are small perturbations to the base flow in the form $q^{\prime}(x, z, t)=\hat{q}(r, t) e^{i m \theta}$. Using these expressions in the ideal MHD equations and neglecting terms of higher orders in perturbations, the linearized equations of the perturbed state are given in the nonconservative form as

$$
\begin{aligned}
& \frac{1}{r} \frac{\partial\left(r \hat{u}_{r}\right)}{\partial r}+\frac{i m}{r} \hat{u}_{\theta}=0 \\
& \stackrel{\circ}{\rho} \frac{\partial \hat{u}_{r}}{\partial t}+\frac{1}{r} \frac{\partial\left(r\left[2 \stackrel{\circ}{\rho} \hat{u}_{r} \stackrel{\circ}{u}_{r}+\hat{p}_{t}-2 \hat{B}_{r} \stackrel{\circ}{B}_{r}\right]\right)}{\partial r}+\frac{i m}{r}\left[\stackrel{\circ}{\rho}\left(\hat{u}_{r} \stackrel{\circ}{u}_{\theta}+\stackrel{\circ}{u}_{r} \hat{u}_{\theta}\right)-\left(\hat{B}_{r} \stackrel{\circ}{B}_{\theta}+\stackrel{\circ}{B}_{r} \hat{B}_{\theta}\right)\right] \\
& =-\frac{1}{r}\left[2 \hat{B}_{\theta} \stackrel{\circ}{B}_{\theta}-2 \hat{u}_{\theta} \stackrel{\circ}{u}_{\theta}-\hat{p}_{t}\right]+\left(\stackrel{\circ}{\rho}_{2}-\stackrel{\circ}{\rho}_{1}\right)[(H(r)-H(r-f)] \Delta V \delta(t), \\
& \stackrel{\circ}{\rho} \frac{\partial \hat{u}_{\theta}}{\partial t}+\frac{1}{r} \frac{\partial\left(r\left[\stackrel{\circ}{\rho}\left(\hat{u}_{r} \stackrel{\circ}{u}_{\theta}+\stackrel{\circ}{u}_{r} \hat{u}_{\theta}\right)-\left(\hat{B}_{r} \stackrel{\circ}{B}_{\theta}+\stackrel{\circ}{B}_{r} \hat{B}_{\theta}\right)\right]\right)}{\partial r}+\frac{i m}{r}\left[2 \stackrel{\circ}{\rho} \hat{u}_{\theta} \stackrel{\circ}{u}_{\theta}+\hat{p}_{t}-2 \hat{B}_{\theta} \stackrel{\circ}{B}_{\theta}\right] \\
& =-\frac{1}{r}\left[\stackrel{\circ}{\rho}\left(\hat{u}_{r} \stackrel{\circ}{u}_{\theta}+\stackrel{\circ}{u}_{r} \hat{u}_{\theta}\right)-\left(\hat{B}_{r} \stackrel{\circ}{B}_{\theta}+\stackrel{\circ}{B}_{r} \hat{B}_{\theta}\right)\right], \\
& \frac{\partial \hat{B}_{r}}{\partial t}+\frac{i m}{r}\left[\stackrel{\circ}{u}_{\theta} \hat{B}_{r}+\hat{u}_{\theta} \stackrel{\circ}{B}_{r}-\left(\stackrel{\circ}{u}_{r} \hat{B}_{\theta}+\hat{u}_{r} \stackrel{\circ}{B}_{\theta}\right)\right]=0, \\
& \frac{\partial \hat{B}_{\theta}}{\partial t}+\frac{1}{r} \frac{\partial r\left[\hat{u}_{r} \stackrel{\circ}{B}_{\theta}+\hat{B}_{\theta} \stackrel{\circ}{u}_{r}-\left(\hat{B}_{r} \stackrel{\circ}{u}_{\theta}+\stackrel{\circ}{B}_{r} \hat{u}_{\theta}\right)\right]}{\partial r}=\frac{-1}{r}\left[\stackrel{\circ}{u}_{\theta} \hat{B}_{r}+\hat{u}_{\theta} \stackrel{\circ}{B}_{r}-\left(\stackrel{\circ}{u}_{r} \hat{B}_{\theta}+\hat{u}_{r} \stackrel{\circ}{B}_{\theta}\right)\right], \\
& \frac{\partial\left(r \hat{B}_{r}\right)}{\partial r}+i m \hat{B}_{\theta}=0
\end{aligned}
$$

where the total pressure is $\hat{p}_{t}=\hat{p}+\hat{B}_{r} \stackrel{\circ}{B}_{r}+\hat{B}_{\theta} \stackrel{\circ}{B}_{\theta}$. Note here, Eq. (3a) is the perturbed solenoidal condition on the velocity, i.e., $\boldsymbol{\nabla} \cdot \mathbf{u}=0$, which signifies the incompressibility condition of the flow.

\section{A. Normal magnetic field}

Expanding the derivatives of Eqs. (3) and using the base flow quantities Eqs. (1), the system Eqs. (3) is simplified as follows:

$$
\begin{aligned}
r \frac{\partial \hat{u}_{r}}{\partial r}+\hat{u}_{r}+i m \hat{u}_{\theta} & =0, \\
\stackrel{\rho}{\rho} \frac{\partial \hat{u}_{r}}{\partial t}+\frac{\partial \hat{p}_{t}}{\partial r}-2 b_{r} \frac{\partial \hat{B}_{r}}{\partial r}-\frac{2 b_{r}}{r} \hat{B}_{r}-\frac{i m b_{r}}{r} \hat{B}_{\theta} & =\left(\stackrel{\circ}{\rho}_{2}-\stackrel{\circ}{\rho}_{1}\right)[(H(r)-H(r-f)] \Delta V \delta(t), \\
\stackrel{\circ}{\rho} \frac{\partial \hat{u}_{\theta}}{\partial t}-b_{r} \frac{\partial \hat{B}_{\theta}}{\partial r}-\frac{2 b_{r}}{r} \hat{B}_{\theta}+\frac{i m}{r} \hat{p}_{t} & =0,
\end{aligned}
$$




$$
\begin{aligned}
\frac{\partial \hat{B}_{r}}{\partial t}+\frac{i m b_{r}}{r} \hat{u}_{\theta} & =0, \\
\frac{\partial \hat{B}_{\theta}}{\partial t}-b_{r} \frac{\partial \hat{u}_{\theta}}{\partial r} & =0, \\
r \frac{\partial \hat{B}_{r}}{\partial r}+\hat{B}_{r}+i m \hat{B}_{\theta} & =0 .
\end{aligned}
$$

The initial conditions are imposed at $t=0^{-}$, just prior to the impulsive acceleration, when the velocity and magnetic field perturbations are zero. The forcing of the perturbations due to the impulse at $t=0^{+}$is nonzero only in the vanishingly small region $r \in\left[R_{0}, f(\theta, t)\right]$. Taking the temporal Laplace transforms (LT) of Eqs. (4) outside the forcing region in each fluid gives the transformed homogeneous equations as

$$
\begin{aligned}
r D U_{j}+U_{j}+i m V_{j} & =0, \\
\stackrel{\rho}{\rho}_{j} s U_{j}+D P_{j}-2 b_{r} D H_{r j}-\frac{2 b_{r}}{r} H_{r j}-\frac{i m b_{r}}{r} H_{\theta j} & =0, \\
\stackrel{\rho}{j}_{j} s V_{j}-b_{r} D H_{\theta j}-\frac{2 b_{r}}{r} H_{\theta j}+\frac{i m}{r} P_{j} & =0, \\
s H_{r j}+\frac{i m b_{r}}{r} V_{j} & =0, \\
s H_{\theta j}-b_{r} D V_{j} & =0, \\
r D H_{r j}+H_{r j}+i m H_{\theta j} & =0,
\end{aligned}
$$

where $U, V, H_{r}, H_{\theta}$, and $P$ are the temporal LT of $\hat{u}_{r}, \hat{u}_{\theta}, \hat{B}_{r}, \hat{B}_{\theta}$ and $\hat{p}_{t}$, respectively. The index $j=1$ and 2 is used for the two fluids, $s$ is the Laplace variable and $D \equiv d / d r$. Then, combining Eqs. (5a)-(5f) gives a single fourth order ODE with a differential operator $\mathcal{L}=\mathcal{L}_{0}+\frac{1}{r} \mathcal{L}_{1}+\frac{1}{r^{2}} \mathcal{L}_{2}$ as follows:

$$
\begin{aligned}
\mathcal{L} U_{j} \equiv & {\left[D^{4}-\left(\frac{s^{2}}{C_{A_{j}}^{2}}+n^{2}\right) D^{2}+\frac{s^{2} n^{2}}{C_{A_{j}}^{2}}\right] U_{j}+\frac{1}{r}\left[4 D^{3}-\left(\frac{3 s^{2}}{C_{A_{j}}^{2}}+2 n^{2}\right) D\right] U_{j} } \\
& +\frac{1}{r^{2}}\left[3 D^{2}-\frac{s^{2}}{C_{A_{j}}^{2}}\right] U_{j}=0 .
\end{aligned}
$$

Here, the differential operator $\mathcal{L}$ is a combination of the exact terms of fourth-order ODE of linear analysis of Wheatley et al. [8] in Cartesian geometry, $\mathcal{L}_{0}$, plus the corrections to the ODE stemming from cylindrical geometry, $\frac{1}{r} \mathcal{L}_{1}+\frac{1}{r^{2}} \mathcal{L}_{2}$. Note that $n=m / r$ refers to the wave number and $C_{A_{j}}=$ $b_{r} / \sqrt{\stackrel{\circ}{\rho}_{j}}$ is the Alfvén wave speed in fluid $j$.

The perturbations must be bounded as $\left|r-R_{0}\right| \rightarrow \infty$ and there are no incoming waves from $\mid r-$ $R_{0} \mid= \pm \infty$, where $\left|r-R_{0}\right|$ is the distance from the interface. We assume that there are no outgoing waves from the origin in the time period of interest, which is also supported by the above mentioned assumption that the radius is large. The matching conditions across the interface $r=R_{0}$, refer to jump conditions of the velocity normal component $\hat{u}_{r}$ which must be continuous. From Eq. (4d) the continuity of the magnetic field $\hat{B}_{r}$ leads to the continuity of $\hat{u}_{\theta}$, which along with Eq. (4a) leads to the continuity of $\frac{d \hat{u}_{r}}{d r}$. Similarly, the continuity of $\hat{B}_{\theta}$ in Eq. (4e) leads to the continuity of $\frac{\hat{u}_{\theta}}{d r}$; this combined with the radial derivative of Eq. (4a) leads to the continuity of $\frac{d^{2} \hat{u}_{r}}{d r^{2}}$ (see Chandrasekhar [21], pp. 458-495). The kinematic condition at the boundary $r=R_{0}$ is

$$
\left[\hat{u}_{r}\right]_{r=R_{0}}=0, \quad\left[\frac{d \hat{u}_{r}}{d r}\right]_{r=R_{0}}=0 \quad \text { and } \quad\left[\frac{d^{2} \hat{u}_{r}}{d r^{2}}\right]_{r=R_{0}}=0 .
$$


Taking Laplace transforms of these variables and using Eqs. (5) to express each in terms of $U_{j}$, these boundary conditions become

$$
[U]_{r=R_{0}}=0, \quad[D U]_{r=R_{0}}=0 \quad \text { and } \quad\left[D^{2} U\right]_{r=R_{0}}=0,
$$

where $[q]_{r=R_{0}}=\left.q_{2}\right|_{r=R_{0}}-\left.q_{1}\right|_{r=R_{0}}$. A dynamic condition is obtained by integrating Eq. (4b) with respect to $r$ from the reference position $r=R_{0}$ to $f$ across its inhomogeneous region, where the term $p=\hat{p}_{t}-2 b_{r} B_{r}$ is the pressure. The integration is contained within fluid 1 , so the resulting equation is written as

$$
\stackrel{\circ}{\rho}_{1} \int_{R_{0}}^{f} \frac{\partial \hat{u}_{r 1}}{\partial t} d r+\hat{p}_{1}(f, \theta, t)-\hat{p}_{1}\left(R_{0}, \theta, t\right)+H . O . T=\int_{R_{0}}^{f}\left(\stackrel{\circ}{\rho}_{2}-\stackrel{\circ}{\rho}_{1}\right) \Delta V \delta(t) d r .
$$

Another assumption is that $\frac{\partial \hat{u}_{r 1}}{\partial t}$ is considered smooth within the inhomogeneous region, so it is approximated by

$$
\left.\stackrel{\circ}{\rho}_{1} \int_{R_{0}}^{f} \frac{\partial \hat{u}_{r 1}}{\partial t} d r \simeq \stackrel{\circ}{\rho}_{1} f \frac{\partial \hat{u}_{r 1}}{\partial t}\right|_{r=R_{0}} .
$$

This term is $O\left(f^{2}\right)$, and thus it can be neglected. Next, the continuity of $\hat{p}$ across the contact discontinuity $r=f$ is expressed as $[\hat{p}]=0$. Assuming $\hat{p}_{1}$ and $\hat{p}_{2}$ are smooth then

$$
\hat{p}_{1}(f, \theta, t)=\hat{p}_{2}(f, \theta, t) \simeq \hat{p}_{2}\left(R_{0}, \theta, t\right) .
$$

Combining Eq. (9) together with Eqs. (10) and (11) and neglecting the higher order terms we get the final form of the dynamic condition:

$$
\hat{p}_{2}\left(R_{0}, \theta, t\right)-\hat{p}_{1}\left(R_{0}, \theta, t\right)=\left(\stackrel{\circ}{\rho}_{2}-\stackrel{\circ}{\rho}_{1}\right) \Delta V \delta(t) e^{i m \theta} .
$$

Taking LT of the previous equation gives the transformed pressure magnitude as follows:

$$
[P]_{r=R_{0}}=\left(\stackrel{\circ}{\rho}_{2}-\stackrel{\circ}{\rho}_{1}\right) \Delta V \eta_{0} .
$$

The solution of the fourth-order ODE (6) is found by numerically by a finite difference approximation. The solution in physical space is then computed using numerical inverse Laplace transform. The details of the numerical procedure are presented in Appendix A.

\section{B. Azimuthal magnetic field}

For this case, we expand the derivatives in Eqs. (3) and use the base flow quantities Eq. (2). The resulting linearized equations are

$$
\begin{aligned}
& r \frac{\partial \hat{u}_{r}}{\partial r}+\hat{u}_{r}+i m \hat{u}_{\theta}=0 \\
& \stackrel{\circ}{\rho} \frac{\partial \hat{u}_{r}}{\partial t}+\frac{\partial \hat{p}_{t}}{\partial r}+\frac{2 b_{t}}{r} \hat{B}_{\theta}-\frac{i m b_{t}}{r} \hat{B}_{r}=\left(\stackrel{\circ}{\rho}_{2}-\stackrel{\circ}{\rho}_{1}\right)[(H(r)-H(r-f)] \Delta V \delta(t), \\
& \stackrel{\partial}{\rho} \frac{\partial \hat{u}_{\theta}}{\partial t}+\frac{i m}{r} \hat{p}_{t}-b_{t} \frac{\partial \hat{B}_{r}}{\partial r}-\frac{2 b_{t}}{r} \hat{B}_{r}-\frac{2 b_{t} i m}{r} \hat{B}_{\theta}=0, \\
& \frac{\partial \hat{B}_{r}}{\partial t}+\frac{i m b_{t}}{r} \hat{u}_{r}=0 \\
& \frac{\partial \hat{B}_{\theta}}{\partial t}+b_{t} \frac{\partial \hat{u}_{r}}{\partial r}=0 \\
& r \frac{\partial \hat{B}_{r}}{\partial r}+\hat{B}_{r}+i m \hat{B}_{\theta}=0 .
\end{aligned}
$$


The initial conditions are imposed at $t=0^{-}$, just before the impulsive acceleration, when the velocity and magnetic field perturbations are zero. Taking the temporal LT of Eqs. (14) outside the forcing region in each fluid gives

$$
\begin{aligned}
r D U_{j}+U_{j}+i m V_{j} & =0, \\
\stackrel{\rho}{\rho}_{j} s U_{j}+D P_{j}+\frac{2 b_{t}}{r} H_{\theta j}-\frac{i m b_{t}}{r} H_{r j} & =0, \\
\stackrel{\rho}{\rho}_{j} s V_{j}-b_{t} D H_{r j}-\frac{2 b_{t}}{r} H_{r j}-\frac{i m b_{t}}{r} H_{\theta j}+\frac{i m}{r} P_{j} & =0, \\
s H_{r j}+\frac{i m b_{t}}{r} U_{j} & =0, \\
s H_{\theta j}+b_{t} D U_{j} & =0, \\
r D H_{r j}+H_{r j}+i m H_{\theta j} & =0 .
\end{aligned}
$$

The Eqs. (15a)-(15f) are combined which gives a second-order ODE of the transformed radial velocity magnitude in each fluid, with $\mathcal{L}=\mathcal{L}_{0}+\frac{1}{r} \mathcal{L}_{1}+\frac{1}{r^{2}} \mathcal{L}_{2}+\frac{1}{r^{4}} \mathcal{L}_{3}$ as

$\mathcal{L} U_{j} \equiv\left[D^{2}-n^{2}\right] U_{j}+\frac{1}{r}\left[3+\frac{C_{A_{j}}^{2} n^{2}}{s^{2}}\right] D U_{j}+\frac{1}{r^{2}}\left[1+\frac{C_{A_{j}}^{2}}{s^{2}}\left(n^{2}+m^{2} D^{2}\right)\right] U_{j}-\frac{1}{r^{4}}\left[\frac{C_{A_{j}}^{2} m^{4}}{s^{2}}\right] U_{j}=0$,

where $C_{A_{j}}=b_{t} / \sqrt{\stackrel{\circ}{\rho}_{j}}$. Also in this case, the differential operator $\mathcal{L}$ is a combination of the secondorder ODE of linear analysis of Wheatley et al. [13], $\mathcal{L}_{0}$ in Cartesian geometry, plus the corrections to the ODE stemming from cylindrical geometry, $\frac{1}{r} \mathcal{L}_{1}+\frac{1}{r^{2}} \mathcal{L}_{2}+\frac{1}{r^{4}} \mathcal{L}_{3}$. The solution is subjected to boundary and matching conditions which are similar to the conditions presented in Sec. III A. The perturbations must be bounded as $\left|r-R_{0}\right| \rightarrow \infty$ and there are no incoming waves from $r= \pm \infty$, where $r$ is distance from $R_{0}$, the jump conditions across the interface:

$$
[U]_{r=R_{0}}=0,[D U]_{r=R_{0}}=0, \text { and }\left[D^{2} U\right]_{r=R_{0}}=0 .
$$

However, the nature of the interface requires further investigation. If the unperturbed interface has no magnetic field lines crossing it and is known as a tangential discontinuity. Only the normal velocity $v$ and $p+\mathbf{B} \cdot \mathbf{B} / 2$ are required to be continuous across a tangential discontinuity (see, e.g., Sutton and Sherman [22]), and these were the only constraints built into the solution of the model problem. Therefore, the last matching condition is obtained by integrating Eq. (14b) with respect to $r$ and following the same process we get the final form of dynamic condition:

$$
\hat{p}_{2}\left(R_{0}, \theta, t\right)-\hat{p}_{1}\left(R_{0}, \theta, t\right)=\left(\stackrel{\circ}{\rho}_{2}-\stackrel{\circ}{\rho}_{1}\right) \Delta V \delta(t) e^{i m \theta} .
$$

Taking LT of the previous equation we get

$$
[P]_{R_{0}=0}=\left(\stackrel{\circ}{\rho}_{2}-\stackrel{\circ}{\rho}_{1}\right) \Delta V \eta_{0} .
$$

The ODE (16) is simplified as follows:

$$
r^{2} D^{2} U_{j}+r\left[\frac{3 s^{2} r^{2}+C_{A_{j}}^{2} m^{2}}{s^{2} r^{2}+C_{A_{j}}^{2} m^{2}}\right] D U_{j}+\left[1-m^{2}\right] U_{j}=0
$$

This equation does permit an analytical solution in terms of hypergeometric functions. This analytical solution then needs to be transformed into the physical domain using inverse Laplace transform, which turns out to be intractable and hence we resort to numerical means to solve the above system. The details of the numerical method are relegated to Appendix A. 


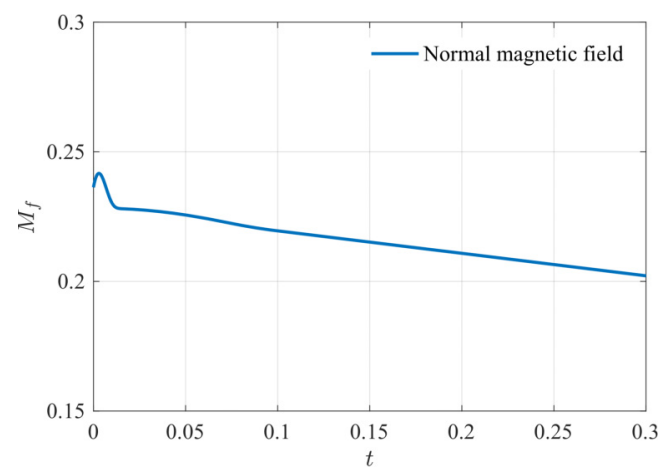

(a)

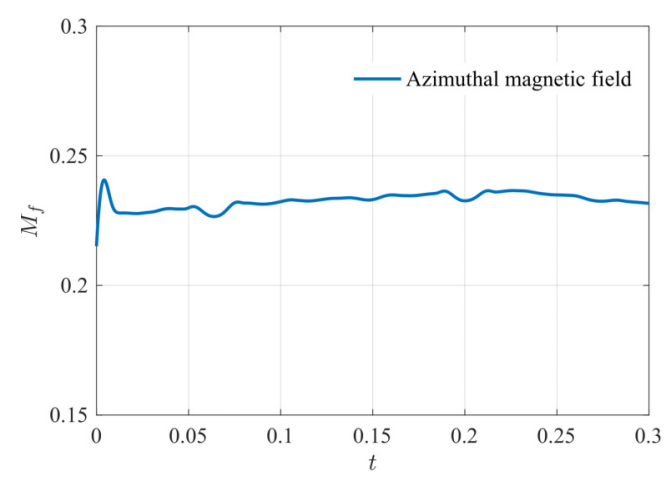

(b)

FIG. 3. The fast Mach number of the perturbed interface $M_{f}$ (a) normal magnetic field and (b) azimuthal magnetic field.

\section{RESULTS AND DISCUSSIONS}

In this section, we present results of the incompressible model and the compressible simulations of MHD RMI in planar and cylindrical geometries. First, we justify the incompressible assumption by examining the Mach number of the flow from numerical compressible MHD simulations. Second, in a planar geometry the numerical result of the incompressible model proposed by Wheatley et al. [8] is presented and our method is verified against their analytical solution. Then, we present the numerical solution of the incompressible models in the cylindrical geometry, and we compare the results with compressible linear simulations of MHD RMI for both cases of normal and azimuthal magnetic fields. These compressible simulations have been introduced and investigated by Bakhsh et al. [20,23]. A uniform grid with 1024 cells is utilized for the numerical solution.

In the shock-driven analysis by Richtmyer [1] after the incident shock interacts with the interface it bifurcates into transmitted and reflected shocks. He suggested that as these two waves travel to a sufficient distance from the interface, the motion of the flow around the interface may be considered as an incompressible one. Here, we show that the flow around the perturbed interface in the linear simulations may be considered incompressible, which then allows for a meaningful comparison between compressible linear numerical simulations and the incompressible models. We examine the ratio of the interface velocity $\stackrel{\circ}{u}_{r}$ to the local fast wave speed which is the fast Mach number:

$$
\begin{aligned}
& M_{f}=\frac{\left|\stackrel{\leftrightarrow}{u}_{r}\right|}{v_{f}}, \quad \text { where } \quad v_{f}=\sqrt{v_{a}^{2}+v_{c}^{2}}, \\
& v_{a}=\frac{\stackrel{\circ}{B}}{\sqrt{\circ}}, \quad \text { and } \quad v_{c}=\sqrt{\frac{\gamma \stackrel{\circ}{\rho}}{\rho}}
\end{aligned}
$$

where $v_{a}$ is the local Alfvén speed and $v_{c}$ is the local sound speed. The assumption for incompressible flows is that the flow remains within a Mach number limit (normally less than 0.3), which is satisfied in our cases for the values of $M_{f}$, as seen in Fig. 3 for the case of (a) normal magnetic field and (b) azimuthal magnetic field for $\beta=16, \rho_{2} / \rho_{1}=3$ and $M=1.25$. Other cases considered later are also in this incompressible regime.

\section{A. Verification of the incompressible model in planar geometry}

The analytical solution of the incompressible model presented by Wheatley et al. [8] is used to verify the numerical solution for same model. In the planar geometry, we use $(x, z)$ coordinates for this model where the impulsive acceleration is in $z$ direction. The linearized initial value problem of 


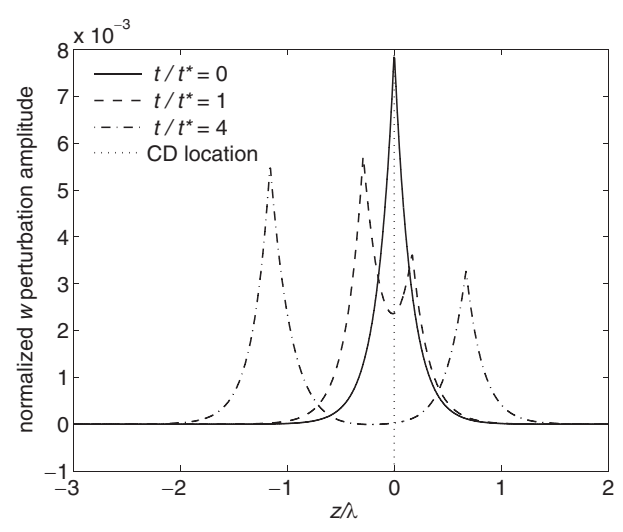

(a)

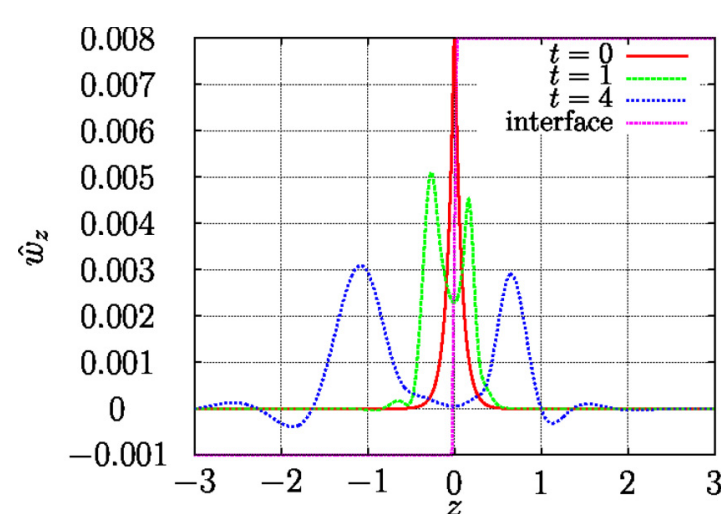

(b)

FIG. 4. Profiles of velocity $\hat{w}$ at $t=0,1,4$ : (a) analytical solution (reproduced from Wheatley et al. [8]), (b) present numerical solution.

the incompressible model was simplified by using LT and they obtained a fourth-order ODE,

$$
D^{4} W_{j}-\left(\frac{s^{2}}{C_{A j}^{2}}+k^{2}\right) D^{2} W_{j}+\frac{s^{2} k^{2}}{C_{A j}^{2}} W_{j}=0,
$$

where $W_{j}$ is the temporal LT of the perturbed horizontal velocity component $\hat{w}, k$ is the wave number and $D=\frac{d}{d z}$. The initial conditions correspond to the case of accelerated interface by shock of strength $M=1.25$ with $\rho_{1}^{+}=1.48372, \rho_{2}^{+}=4.43159, \Delta V=0.319117, \eta_{0}=0.00799$ and $\beta=16$. The boundary conditions used to solve this equation are clarified in Ref. [8]. Figure 4 shows the profiles of $\hat{w}(z, t)$ at various times (a) the analytical solution of Wheatley et al. [8] (b) present numerical solution. At $z=0$ the value of $\hat{w}(z, t)$ represents the growth rate of the interface, as $t$ increases the maximum velocity peaks coincide with Alfvén fronts and propagate away from the interface, therefore the growth rate decays to zero. The analytical solution is shown in Fig. 4(a) from Ref. [8] where there are two sharp cusps representing Alfvén fronts. The comparison between numerical results and the previous analytical results are quite reasonable although, dispersion errors stemming from our purely central difference schemes affect the sharpness of the cusps and we also get some undershoots. We conclude that our numerical solutions is able to adequately reproduce the analytical solution. Next, the results of the incompressible model in cylindrical geometry are presented and compared with the numerical simulations of the compressible MHD RMI which have been previously discussed in detail in Refs. [20,23].

\section{B. Normal magnetic field in cylindrical geometry}

The geometry considered for linear simulations is presented in Fig. 1(a). In the reference shockdriven linear simulations, the ratio of specific heats is set to $\gamma=5 / 3$, the initial density ratio is $\stackrel{\circ}{\rho}_{2} / \stackrel{\rho}{\rho}_{1}=3$, the pressure upstream of the shock is $\stackrel{\circ}{p}=1$, the shock Mach number $M=1.25$, and the normal magnetic field is characterized by $\beta=16$. The behavior of the present incompressible model is evaluated using the values that corresponds to post-shock quantities. Post-shock densities $\rho_{1}^{+}=1.48372, \rho_{2}^{+}=4.43159$, the magnitude of the impulse is set to the interface velocity in the shock-driven problem as $\Delta V=0.319117$ with magnitude of magnetic field $\beta=16$. The solution of Eq. (6) is obtained numerically using LU decomposition, then taking the numerical ILT to evaluate the perturbed velocity in each fluid. The profile of $\hat{u}_{r}$ is plotted at different times in Fig. 5 where the values at $R_{0}=1$ are the growth rate of the interface. As in the previous observation in the incompressible simulations of MHD RMI in the presence of normal magnetic field in cylindrical 


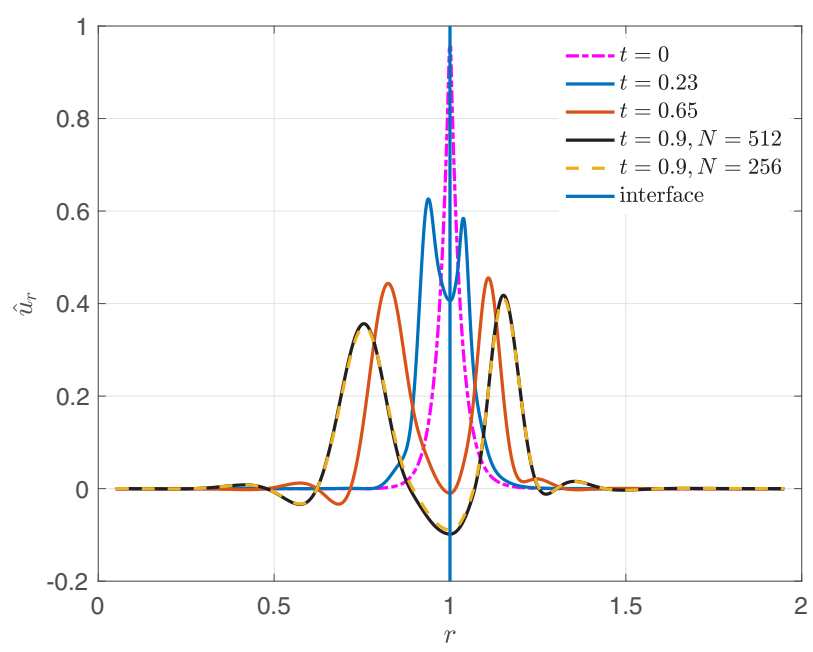

FIG. 5. Profiles of velocity $\hat{u}_{r}$, using numerical ILT at $t=0,0.23,0.65,0.9$, for $\rho_{1}^{+}=1.4837, \rho_{2}^{+}=$ 4.4315, $\Delta V=0.319117, \beta=16$, corresponding to $M=1.25$.

geometry ([20] Fig. 13(f)), we see two dominant Alfvén fronts coincide with the vortex sheets ([20] Fig. 13(e)). These Alfvén fronts are seen in the incompressible model solution which transport the vorticity away from the interface position. They propagate with the local Alfvén speed. Moreover, we added an additional plot for $t=0.9$ in Fig. 5 corresponding to a coarser mesh resolution $(N=$ 256) which is almost identical to the current mesh choice $N=512$. The growth rate of the interface after the initial increase, decays to value less than zero. For different wave numbers, $m=32,128$ and $\beta=16$ the results of scaled growth rate of the interface are compared with the results of linear simulations and presented in Fig. 6: the model matches the simulations at the early time and the large wave number $m=128$ shows a better agreement than the lower one. It is known in converging geometry that as the interface converges to the origin, it decelerates. This deceleration effect is not considered in the incompressible analysis because it assumes a constant impulsive speed of the shocked contact discontinuity. As a result, the RTI phase of the instability is not captured by the model. Nevertheless, it shows the decaying growth rate behavior which represents the stabilizing effect of the magnetic field on RMI.

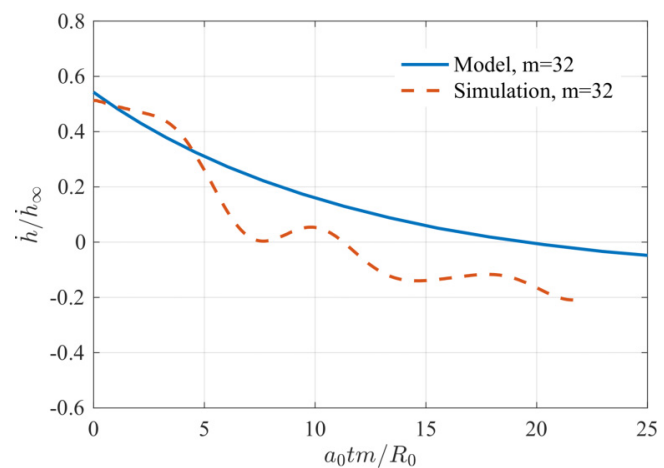

(a)

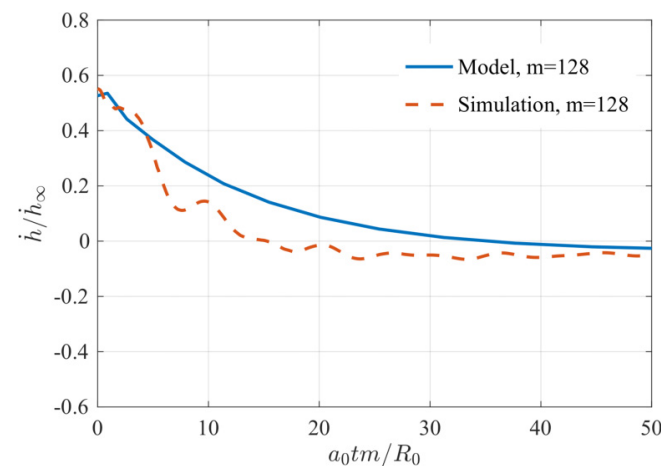

(b)

FIG. 6. Comparison of scaled growth rate between the incompressible model and the linear simulations for the normal field case, (a) $m=32, \beta=16$, (b) $m=128, \beta=16$. 


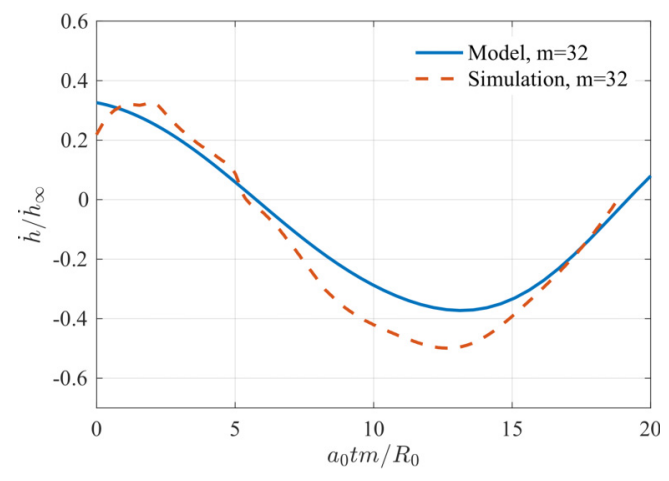

(a)

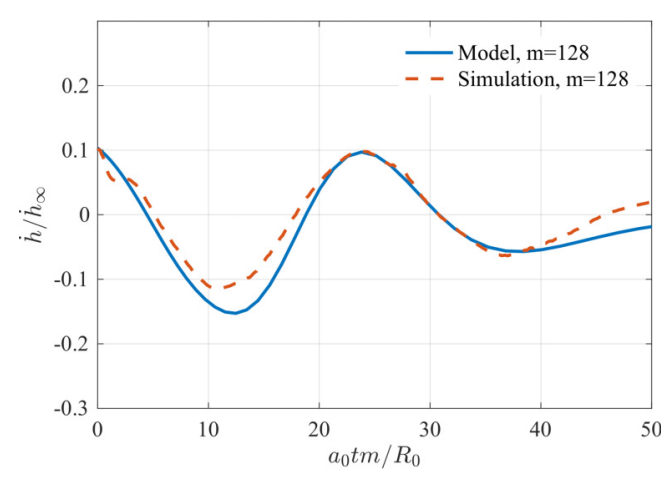

(b)

FIG. 7. Comparison of scaled growth rate between impulsive model and numerical simulations for the azimuthal field case, (a) $m=32, \beta=16$, (b) $m=128, \beta=16$.

\section{Azimuthal magnetic field in cylindrical geometry}

In the presence of an azimuthal magnetic field, Figs. 2(a) and 2(b), shows the geometry of compressible linear simulations and the incompressible model for the RMI, respectively. The behavior of the compressible simulations and the impulsively perturbed interface are examined using the initial values that presented in Sec. IV B. For the case of the incompressible model, the second-order ODE (16) is solved numerically. The results pertaining to the growth rate of the perturbed interface and compared with linear simulation as shown in Fig. 7 for wave numbers $m=32,128$ and $\beta=16$. For large $m$, the model shows better results because it reproduces the right frequency and magnitude of the amplitude of the interface and matches the linear simulation results at early scaled time. The investigation of compressible MHD RMI in the presence of an azimuthal magnetic field in cylindrical geometry [23] explained the dominant physical effect was the transport of vorticity by waves propagating at the local Alfvén speed, parallel and antiparallel to the interface. The interference of these waves, with alternating phase causes constructive and destructive interference and vorticity patterns alternate with changing signs. The net result is that the growth rate of the interface perturbation oscillates from positive to negative. As mentioned earlier, the process is very similar to that seen in planar nonlinear simulations of Wheatley et al.(see Ref. [13], Fig. 6). An obvious effect of the azimuthal seed field is that it suppresses the instability of the interface. Next, we briefly investigate the influence of field strength, Atwood ratio and shock strength. The parameters investigated are summarized in Table I.

\section{Effect of increasing magnetic field}

In this section, the effect of increasing magnetic field is investigated by comparing the $\beta=16$ with the $\beta=4$ (stronger field) case, with the remaining parameters identical to the case of azimuthal

TABLE I. Different Atwood numbers $A$, magnetic strength $\beta$, and wave numbers $m$ corresponding to different $M=1.1,1.25,2$.

\begin{tabular}{lccc}
\hline \hline$M$ & $A$ & $\beta$ & $m$ \\
\hline 1.1 & $1 / 2$ & 16 & 128 \\
1.25 & $1 / 9$ & 16 & 128 \\
& $1 / 3$ & 16 & 128 \\
& $1 / 2$ & 4,16 & 32,128 \\
2 & $1 / 2$ & 16 & 128 \\
\hline \hline
\end{tabular}




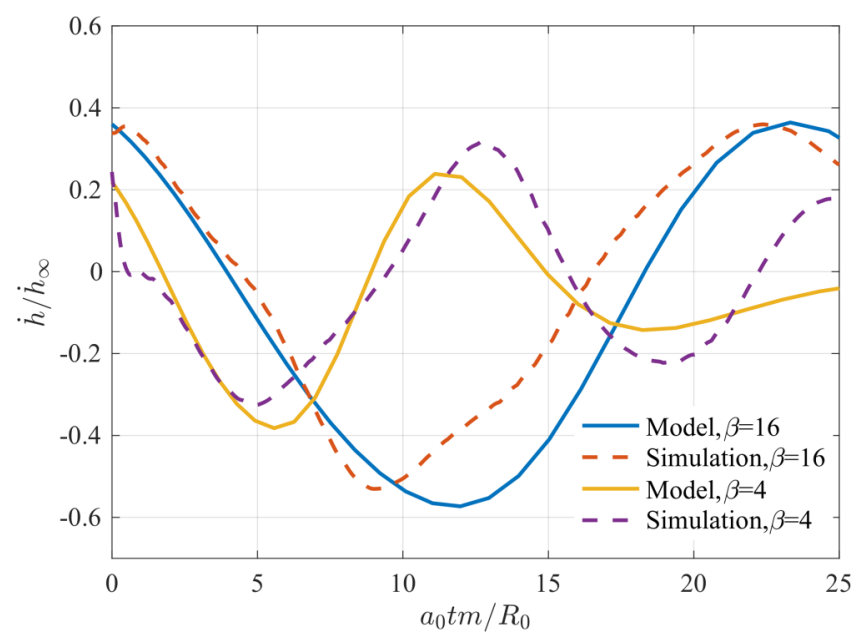

FIG. 8. Growth rate histories from the shock-driven simulations and the linear model for $\rho_{2} / \rho_{1}=3, m=$ $128, M=1.25$ and different field strengths $\beta=16,4$.

magnetic field discussed above. The interface growth rate histories from the $\beta=16$ and $\beta=4$ simulations are compared with our model predictions. It can be seen that, increasing magnetic field strength restricts the amplitude of the perturbed interface and increases the frequency of oscillations. This is explained by the fast propagation of the vorticity carrying waves in the vicinity of the interface, causing more rapid but smaller amplitude oscillations. The incompressible model agrees well with the amplitude with small phase differences for early times. These results are presented in Fig. 8.

\section{E. Effect of increasing Atwood number}

Here, we investigate the effect of fluid densities on the MHD RMI for density ratios $\left(\rho_{2} / \rho_{1}\right)$ of $5 / 4,2$, and 3 , corresponding to Atwood numbers $(A)$ of $1 / 9,1 / 3$, and $1 / 2$, respectively, for $m=128$ and $\beta=16$ with the reference case of $M=1.25$. Figure 9 shows the normalized $\dot{h}$ histories from the relevant simulations along with the incompressible model predictions. The Alfvén speed in the heavy fluid decreases like $\rho_{2}^{-1 / 2}$ as the density increases, which causes the observed decrease in the frequency $\omega$. The model can predict this decrease accurately at early times, for all three simulations. It also shows that the normalized growth rates decrease with increasing $A$. The model predicts similar accuracy for lower $A$ considered here at the first oscillation period. However, for higher $\mathrm{A}$, the interface oscillations become more rapidly dissipated. This can be explained as follows: the vorticity on either side of the interface is transported by the effect of waves propagating at the local Alfvén speed, parallel and antiparallel to the interface. The interference of these waves alternating phase causes constructive and destructive interference, and vorticity patterns alternate with changing sign [23].

\section{F. Effect of increasing shock strength}

The effects of increasing shock strength on the MHD RMI is determined as follow. Linear compressible simulations have been carried out for incident shock sonic Mach numbers of $M=$ 1.1, 1.25 and $M=2$, with the remaining parameters: $\rho_{2} / \rho_{1}=3, \beta=16$ and $m=128$. In Fig. 10 the interface growth rate histories from these simulations are compared to the model predictions using corresponding post-shock quantities and the impulse magnitude at the interface for each Mach number. As shock strength increases, both the frequency and amplitude of the interface oscillations increase and appear to scale as predicted by the model. At higher Mach numbers, the greater shock 


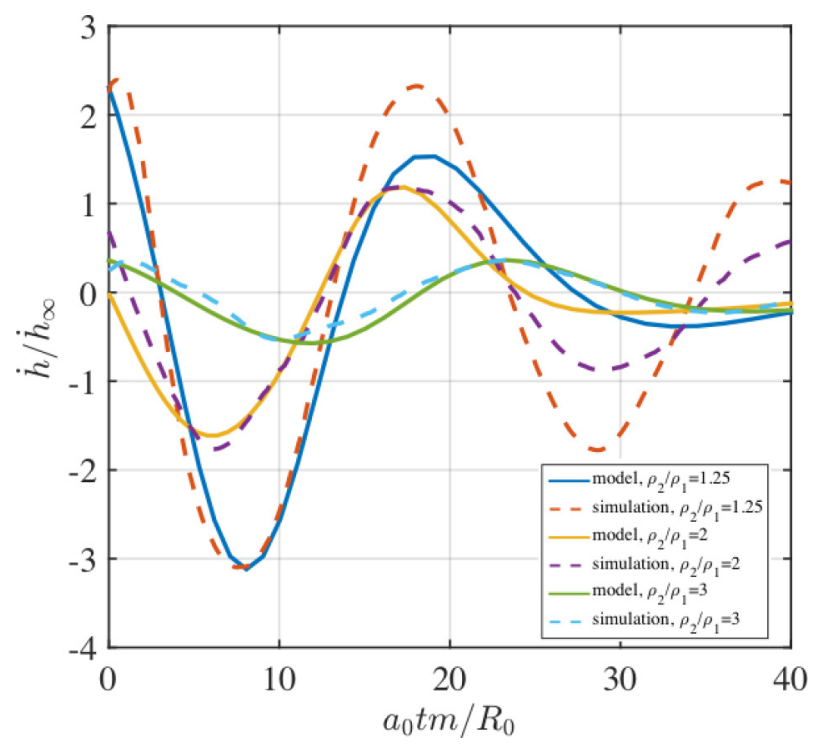

FIG. 9. Growth rate histories from the shock-driven simulations and the linear model for $\rho_{2} / \rho_{1}=$ $5 / 4,2,3$, with $m=128, M=1.25$, and $\beta=16$.

compression causes both $\rho$ and $B$ at the interface to increase with $M$. As the oscillation frequency scales with $B / \sqrt{\rho}$, this results in a sublinear increase in $\omega$ with $M$. The increasing of the growth rate with $\mathrm{M}$ is a result of the increasing velocity $\Delta V$ imparted to the interface. This overwhelms the damping effect of the increase in $\omega \propto B / \sqrt{\rho}$. The large initial spike in for $M=2$, shown in Fig. 10, is not captured by the model. This is likely due to the growth caused by the vorticity transported by the transmitted and reflected fast shocks, similar behavior was observed in Wheatley et al. [13], which is only significant at early times when these shocks are close to the interface.

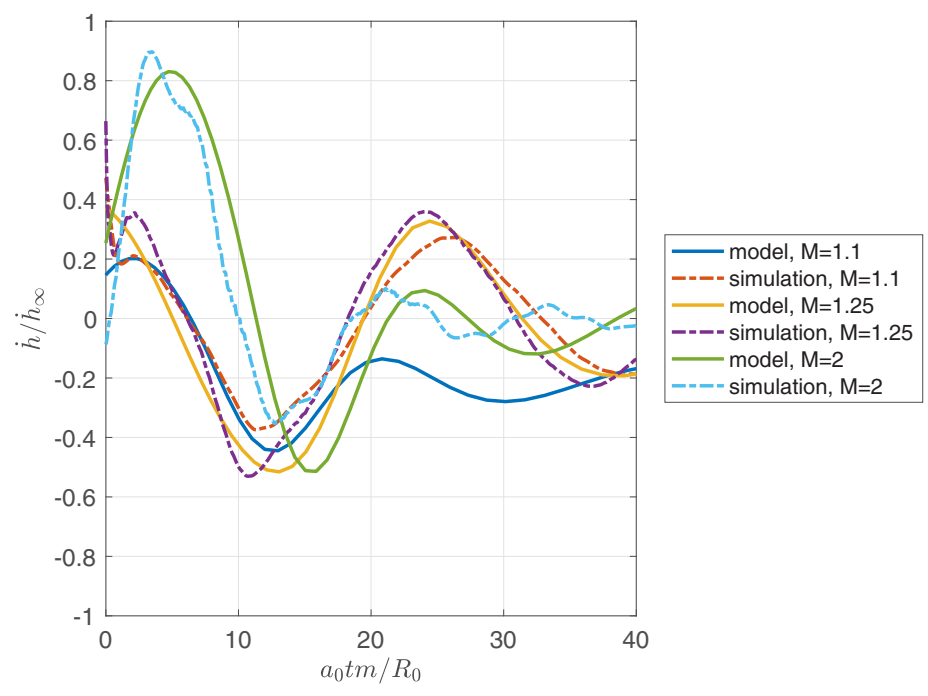

FIG. 10. Growth rate histories from the shock-driven simulations and the linear model for $\rho_{2} / \rho_{1}=3, m=$ $128, \beta=16$ and varying shock strength $M=1.1,1.25,2$. 


\section{CONCLUSION}

The application of incompressible models (generally cast as an "impulse model") have a long history in RMI flows. In the present work, the MHD Richtmyer-Meshkov instability have been investigated for two cases of initial magnetic field configurations in cylindrical geometry: in one case, the magnetic field is parallel to the acceleration, i.e., normal to the interface; whereas in the second case the magnetic field is initially parallel to the interface. The behavior of an impulsively accelerated interface under the assumption of incompressibility is examined. The analysis performed is a linear one by adopting an initial value problem approach to the stability analysis. A numerical solver was developed to obtain complete solution of the problem. For the case of normal magnetic field we found that two Alfvén fronts transport vorticity away from the interface position. After the initial increase the growth rate of the interface, it decays to value less than zero then it slightly increases. For the case of azimuthal magnetic field, after impulsive acceleration of the interface, the emerging vorticity breaks up into waves traveling parallel and antiparallel to the magnetic field. The interference as these waves propagate with alternating phase causing the perturbation growth rate of the interface to oscillate in time. In the analysis, ODEs governing the Laplace transformed velocity at the interface are derived. For the normal (respectively, azimuthal) field case the governing is a fourth-order (respectively, second-order) ODE. The ODEs are expressed using a sum of operators where the leading operator corresponds to the Cartesian planar case. The ODEs are numerically solved using finite differences, and the novel application of a numerical inverse Laplace transform, to obtain the results in the physical domain. For the various cases, examined the incompressible model agrees well with linear compressible simulations (which in turn have been shown in previous work to compare well with nonlinear simulations provided the ratio of the initial amplitude to wavelength is small). We also presented comparisons between linear compressible simulations and the incompressible models by investigating changes in the strength of the field, Atwood ratio, and strength of the impulse. While an exhaustive survey of the parameter space was not performed, these investigations show that the incompressible models reproduced the amplitude and frequency of the growth rate at early times. We simply add here that the incompressible model calculations were relatively computationally very inexpensive and often by several order of magnitude less time consuming that corresponding nonlinear simulations. The present research can be further extended in many aspects: an incompressible model for an impulsively accelerated interface with a time dependent velocity to capture the RTI phase; extending the linear stability analysis to spherical geometry; simulating flows with multiple interfaces; applying other magnetic field configurations and investigating the nonlinear development of these flows.

\section{ACKNOWLEDGMENT}

The research reported in this publication was supported by funding from King Abdullah University of Science and Technology (KAUST), under Award No. 2162.

\section{APPENDIX}

\section{Numerical method}

In this section, we introduce a numerical method to solve the fourth-order ODE [similarly the second-order ODE (16)]:

$$
\begin{aligned}
\mathcal{L} U_{j} \equiv & {\left[D^{4}-\left(\frac{s^{2}}{C_{A_{j}}^{2}}+n^{2}\right) D^{2}+\frac{s^{2} n^{2}}{C_{A_{j}}^{2}}\right] U_{j}+\frac{1}{r}\left[4 D^{3}-\left(\frac{3 s^{2}}{C_{A_{j}}^{2}}+2 n^{2}\right) D\right] U_{j} } \\
& +\frac{1}{r^{2}}\left[3 D^{2}-\frac{s^{2}}{C_{A_{j}}^{2}}\right] U_{j}=0 .
\end{aligned}
$$


The radial domain is confined in the interval $\left[r_{l}, r_{r}\right]$. For the spatial discretization, we use a second order central difference scheme of $2 N+1$ grid points that are equally spaced as $r_{i}=r_{l}+i \Delta r$, where $\Delta r$ is the cell width, $i=-N,-N+1, \ldots,-1,0,1,2, \ldots, N$ and $N \in Z^{+}$. The solution vector $U_{j}\left(r_{i}\right) \simeq U_{i, j}$ represents the velocity magnitude in each fluid (subscript $j=1,2$ ). The spatial discretization of the derivatives are listed as

$$
\begin{aligned}
D^{4} U_{i, j} & =\frac{U_{i+2, j}-4 U_{i+1, j}+6 U_{i, j}-4 U_{i-1, j}+U_{i-2, j}}{\Delta r^{4}}, \\
D^{3} U_{i, j} & =\frac{U_{i+2, j}-2 U_{i+1, j}+2 U_{i-1, j}-U_{i-2, j}}{2 \Delta r^{3}}, \\
D^{2} U_{i, j} & =\frac{U_{i+1, j}-2 U_{i, j}+U_{i-1, j}}{\Delta r^{2}}, \\
D U_{i, j} & =\frac{U_{i+1, j}-U_{i-1, j}}{2 \Delta r} .
\end{aligned}
$$

The kinematic conditions Eqs. (8) across the interface, $r=R_{0}$, are applied at the node $i=0$. They are discretized using the one-sided finite difference of first order accuracy as follows:

$$
\begin{aligned}
U_{0^{-}, 2} & =U_{0^{+}, 1}, \\
D U_{0^{-}, 2} & =\frac{U_{0,2}-U_{-1,2}}{\Delta r}, \quad D U_{0^{+}, 1}=\frac{U_{1,1}-U_{0,1}}{\Delta r}, \\
D^{2} U_{0^{-}, 2} & =\frac{U_{-2,2}-2 U_{-1,2}+U_{0,2}}{\Delta r^{2}}, \quad D^{2} U_{0^{+}, 1}=\frac{U_{0,1}-2 U_{1,1}+U_{2,1}}{\Delta r^{2}} .
\end{aligned}
$$

The numerical implementation of the pressure continuity is performed by combining Eq. (5b) with Eq. (5f), so we get

$$
\stackrel{\circ}{\rho}_{j} s U_{j}+D P_{j}=0 .
$$

The trapezoidal rule is used to approximate the integration of Eq. (A3) with respect to $r$ at the interface position $r=R_{0}$ in the interval $\left[r_{l}, r_{r}\right]$ as

$$
\left.P_{j}\right|_{r=R_{0}}=-\stackrel{\circ}{\rho}_{j} s \int_{r_{l}}^{r_{r}} U_{j} d r=-\stackrel{\circ}{\rho}_{j} s \Delta r\left(\frac{U_{-N, 2}}{2}+\sum_{i=-N+1}^{N-1} U_{i, j}+\frac{U_{N, 1}}{2}\right) .
$$

Then, we express this equation in each fluid as follows:

$$
\begin{aligned}
& \left.P_{2}\right|_{r=R_{0}^{-}}=-\stackrel{\circ}{\rho}_{2} s \Delta r\left(\frac{U_{-N, 2}}{2}+\sum_{i=-N+1}^{-1} U_{i, 2}+\frac{U_{0,2}}{2}\right), \\
& \left.P_{1}\right|_{r=R_{0}^{+}}=-\stackrel{\circ}{\rho}_{1} s \Delta r\left(\frac{U_{0,1}}{2}+\sum_{i=1}^{N-1} U_{i, 1}+\frac{U_{N, 1}}{2}\right) .
\end{aligned}
$$

Substituting these equations into Eq. (13), we get the numerical jump condition at the interface. Therefore, combining the previous discretized fourth-order ODE together with the boundary conditions allows us to build the following linear system:

$$
\boldsymbol{A U}=\boldsymbol{R},
$$

where $\boldsymbol{A}$ is the coefficient matrix, $\boldsymbol{U}$ is the solution vector and $\boldsymbol{R}$ is the RHS that includes the impulsive velocity at the interface. The solution of previous system is obtained numerically using LU decomposition.

Next, we need to obtain the solution in the physical domain. For this step, the numerical inversion of the Laplace transform (ILT) is obtained by Gaver-Stehfest method [24-26]. The Gaver-Stehfest 


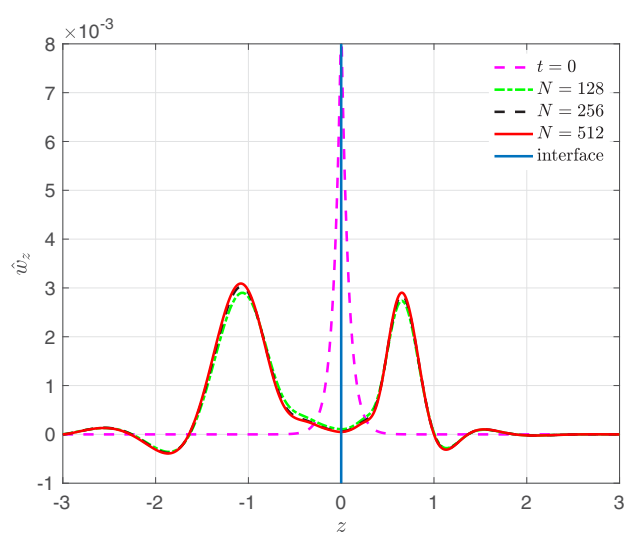

(a)

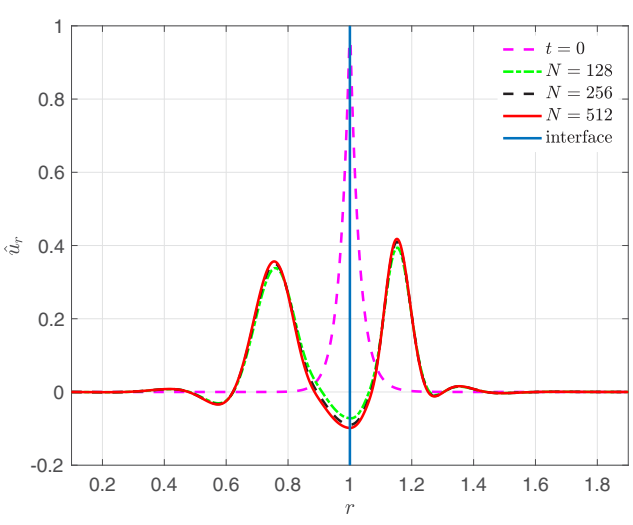

(b)

FIG. 11. Convergence test at different resolutions for the planar and cylindrical geometry cases. The number of mesh points $N$ is varied keeping $M=16$ fixed. (a) Planar geometry solution $\hat{w}_{z}$ at times $t=0,4$ and (b) Cylindrial geometry solution $\hat{u}_{r}$ at times $t=0,0.9$.

technique was reviewed in details by Jacquot et al. [27]. This method is based on deforming the contour in the Bromwich integral to enclose all the singularities of the operator $\mathcal{L}$ inside it. Then, applying the residual theorem to obtain ILT of $\boldsymbol{U}(r, s)$. This integration is performed along the vertical line $s=\alpha$ in the complex plane. The number $\alpha \in \Re$ is chosen so that the line $s=\alpha$ lies in the right of all singularities of the contour. This method approximates the solution of $\hat{u}_{r}(r, t)$ by weighted summation at specific time $t$, given as

$$
\hat{u}_{r}(r, t) \approx \frac{\ln 2}{t} \sum_{l=1}^{M} w_{l} \boldsymbol{U}\left(r, s_{l}\right)
$$

where $l=1,2, \ldots, M, M \in Z^{+}$, the expression of $s_{l}=\frac{l \ln 2}{t}$, and the coefficients $w_{l}$ can be easily computed as

$$
w_{l}=(-1)^{(M / 2)+l} \sum_{k=\left\lfloor\frac{1}{2}(1+l)\right\rfloor}^{\min (l, M / 2)} \frac{2 k ! k^{(M / 2)}}{(M / 2-k) ! k !(k-1) !(l-k) !(2 k-l) !} .
$$

\section{Convergence tests}

Here we present convergence tests for the numerical method presented in Appendix A. The convergence tests are carried out in two ways: in one we vary the number of mesh points $N$ on either side of the interface in the radial domain keeping the number of weights $[M=16$ in Eq. (A7)]; in the other tests we keep the number of the mesh points fixed $(N=512)$ while we vary the number of weights $M$ in the inverse Laplace transform.

Figure 11 shows the results of varying $N$ for the planar and cylindrical geometries at times $t=4,0.9$ for the profiles of $\hat{w}_{z}$ and $\hat{u}_{r}$, respectively. We use $N=128,256$ and $N=512$ points. These figures show almost identical results for both mesh points $N=256$ and $N=512$ cases for each geometry.

Next, in Fig. 12 we plot the error by computing the difference between the analytical solution [shown in Fig. 4(a)] and the numerical solution at time $t=4$ for the planar case. We observe that as the mesh resolution increased the error decreases which implies that the velocity profile $\hat{w}_{z}$ is converging. The order of accuracy is 1.833 , which is reasonably close to second order accuracy. We do not have an analytical solution for the cylindrical case, but since we employ second order finite 


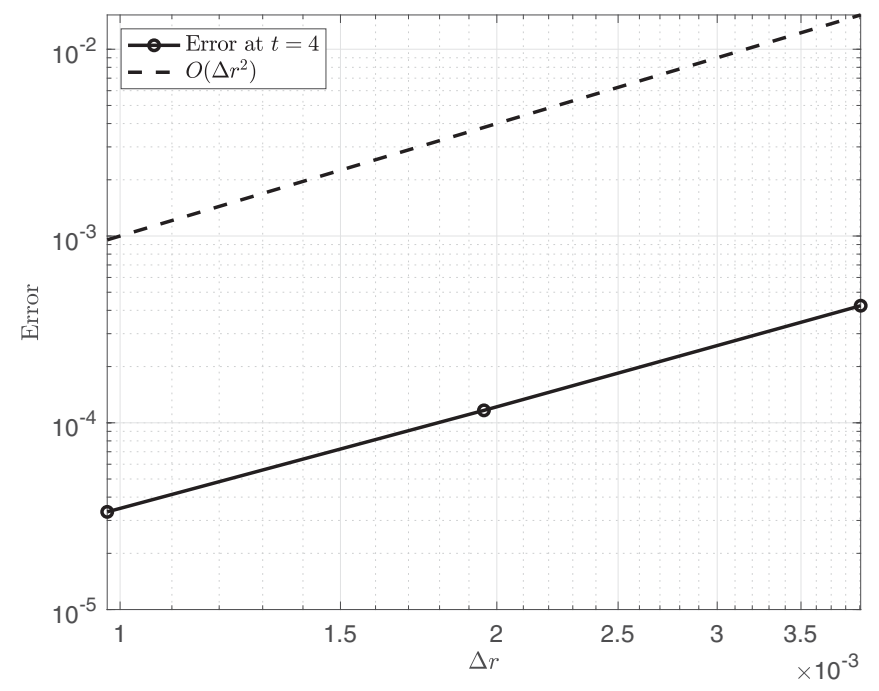

FIG. 12. Error in the numerical solution at time $t=4$ for different mesh resolutions in the numerical inverse Laplace transform in planar geometry.

difference for both the planar and cylindrical cases, we expect a similar order of accuracy for the cylindrical case.

For the second set of convergence test we keep the number of mesh points fixed $(N=512)$ and vary the number of weights $(M)$ in the ILT. Figure 13 shows the results of varying $M$. For both planar and cylindrical cases, increasing the number of weights $M$ shows the difference decreasing between successive increases of $M$.

Varying the number of weights, we plot the error by computing the difference between the numerical result and the analytical for the planar case in Fig. 14. It shows that as the number of weights increases the error decreases indicating convergence. We note here that further that computing weights for $M>24$ with 64-bit precision is not possible. Hence we are limited to the

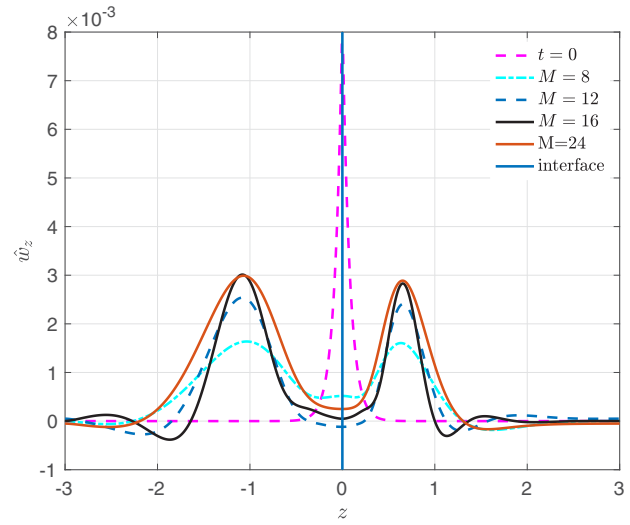

(a)

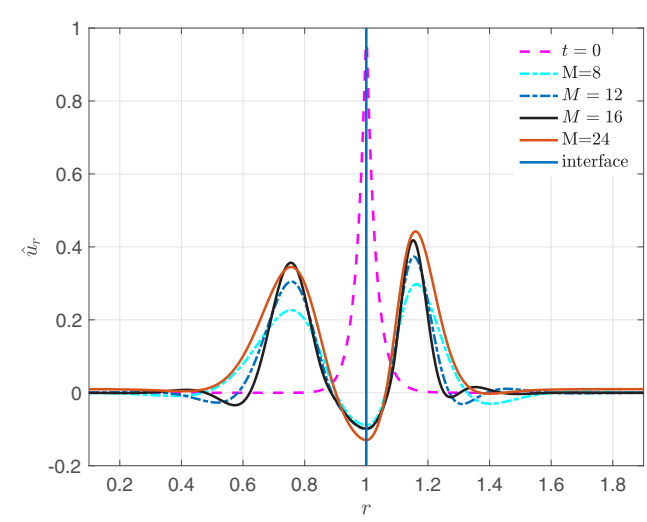

(b)

FIG. 13. Convergence test at different resolutions for the planar and cylindrical geometry cases. The number of mesh points $N=512$ is fixed while $M$ is varied. (a) Planar geometry solution $\hat{w}_{z}$ at times $t=0,4$ and (b) Cylindrial geometry solution $\hat{u}_{r}$ at times $t=0,0.9$. 


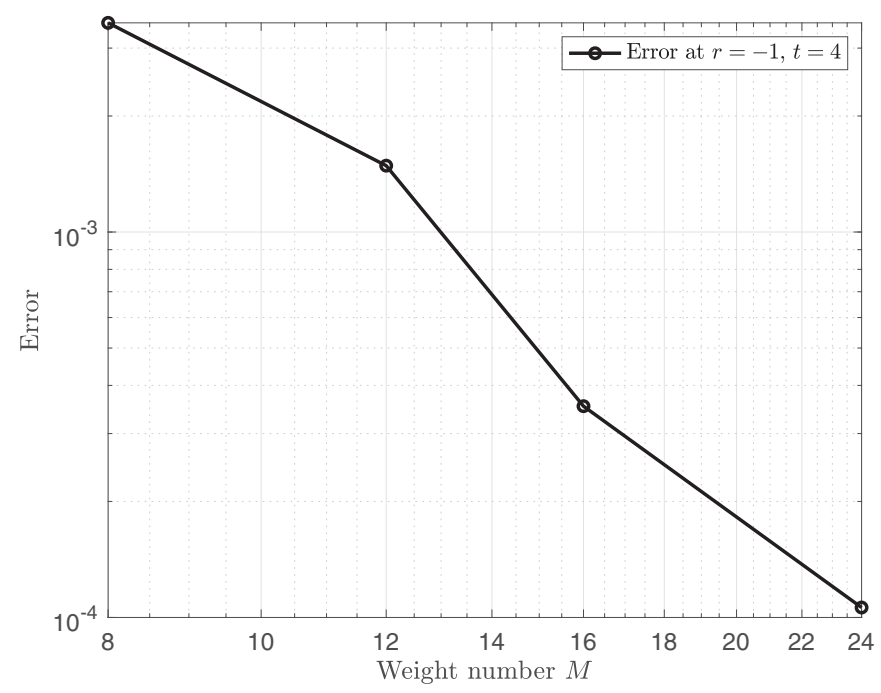

FIG. 14. Error in the numerical results at time $t=4$ with different number of weights in the numerical inverse Laplace transform for planar geometry.

maximum value of $M$ to 24 . We have not explored higher precision arithmetic for a larger number of weights for the numerical inverse Laplace transform algorithm.

[1] R. D. Richtmyer, Taylor instability in shock acceleration of compressible fluids, Commun. Pure Appl. Math. 13, 297 (1960).

[2] E. E. Meshkov, Instability of the interface of two gases accelerated by a shock wave, Fluid Dynamics 4, 101 (1969).

[3] D. Arnett, The role of mixing in astrophysics, Astrophys. J. Suppl. Ser. 127, 213 (2000).

[4] J. Yang, T. Kubota, and E. E. Zukoski, Applications of shock-induced mixing to supersonic combustion, AIAA J. 31, 854 (1993).

[5] M. Brouillette, The Richtmyer-Meshkov instability, Annu. Rev. Fluid Mech. 34, 445 (2002).

[6] G. Fraley, Rayleigh-Taylor stability for a normal shock wave-density discontinuity interaction, Phys. Fluids 29, 376 (1986).

[7] R. Samtaney, Suppression of the Richtmyer-Meshkov instability in the presence of a magnetic field, Phys. Fluids 15, L53 (2003).

[8] V. Wheatley, D. I. Pullin, and R. Samtaney, Stability of an Impulsively Accelerated Density Interface in Magnetohydrodynamics, Phys. Rev. Lett. 95, 125002 (2005).

[9] V. Wheatley, R. Samtaney, and D. I. Pullin, The Richtmyer-Meshkov instability in magnetohydrodynamics, Phys. Fluids 21, 082102 (2009).

[10] Z. Qiu, Z. Wu, J. Cao, and D. Li, Effects of transverse magnetic field and viscosity on the RichtmyerMeshkov instability, Phys. Plasmas 15, 42305 (2008).

[11] J. Cao, Z. Wu, H. Ren, and D. Li, Effects of shear flow and transverse magnetic field on RichtmyerMeshkov instability, Phys. Plasmas 15, 042102 (2008).

[12] Y. Levy, S. Jaouen, and B. Canaud, Numerical investigation of magnetic Richtmyer-Meshkov instability, Laser Part. Beams 30, 415 (2012).

[13] V. Wheatley, R. Samtaney, D. I. Pullin, and R. M. Gehre, The transverse field Richtmyer-Meshkov instability in magnetohydrodynamics, Phys. Fluids 26, 016102 (2014). 
[14] Q. Zhang and M. J. Graham, A numerical study of Richtmyer-Meshkov instability driven by cylindrical shocks, Phys. Fluids 10, 974 (1998).

[15] M. S. Plesset, On the stability of fluid flows with spherical symmetry, J. Appl. Phys. 25, 96 (1954).

[16] K. O. Mikaelian, Rayleigh-Taylor and Richtmyer-Meshkov instabilities and mixing in stratified spherical shells, Phys. Rev. A 42, 3400 (1990).

[17] K. O. Mikaelian, Rayleigh-Taylor and Richtmyer-Meshkov instabilities and mixing in stratified cylindrical shells, Phys. Fluids 17, 094105 (2005).

[18] M. Lombardini and D. I. Pullin, Small-amplitude perturbations in the three-dimensional cylindrical Richtmyer-Meshkov instability, Phys. Fluids 21, 114103 (2009).

[19] M. Brouillette and R. Bonazza, Experiments on the Richtmyer-Meshkov instability: Wall effects and wave phenomena, Phys. Fluids 11, 1127 (1999).

[20] A. Bakhsh, S. Gao, R. Samtaney, and V. Wheatley, Linear simulations of the cylindrical RichtmyerMeshkov instability in magnetohydrodynamics, Phys. Fluids 28, 034106 (2016).

[21] S. Chandrasekhar, Hydrodynamic and Hydromagnetic Stability, Dover Classics of Science and Mathematics (Dover Publications, New York, 1981).

[22] A. Sherman and G. W. Sutton, Engineering Magnetohydrodynamics (McGraw-Hill, New York, 1965).

[23] A. Bakhsh and R. Samtaney, Linear analysis of converging Richtmyer-Meshkov instability in the presence of an azimuthal magnetic field, J. Fluids Eng. 140, 050901 (2017).

[24] A. Davies, The solution of differential equations using numerical Laplace transforms, Int. J. Math. Edu. Sci. Technol. 30, 65 (1999).

[25] A. Kuznetsov, On the convergence of the Gaver-Stehfest Algorithm, SIAM J. Numer. Anal. 51, 2984 (2013).

[26] W. Abate and J. Whitt, A unified framework for numerically inverting laplace transforms, INFORMS J. Comput. 18, 408 (2006).

[27] R. G. Jacquot, J. W. Steadman, and C. N. Rhodine, The Gaver-Stehfest algorithm for approximate inversion of Laplace transforms, IEEE Circ. Syst. Mag. 5, 4 (1983). 